\title{
Preparation and Ordered Self-Assembly of Nano-Pd-Ga/PMMA by Ultrasonic
}

\author{
Xu Guo Cai, DAI Ming Hu, JI Xiao Li, Zhang Xiao Mei, and Xing Hong Long \\ School of Chemical Engineering, Anhui University of Science and Technology, Huainan 232001, China \\ Correspondence should be addressed to Xu Guo Cai, gcxu@aust.edu.cn \\ Received 12 May 2010; Accepted 20 June 2010 \\ Academic Editor: Quanqin Dai \\ Copyright () 2011 Xu Guo Cai et al. This is an open access article distributed under the Creative Commons Attribution License, \\ which permits unrestricted use, distribution, and reproduction in any medium, provided the original work is properly cited.
}

\begin{abstract}
Nano-Ga-Pd/poly methyl methacrylate (PMMA) composite materials were prepared with the palladium chloride solution containing metal gallium, MMA as monomer, and sodium dodecyl sulfate (SDS) as emulsifier without initiatoror reducer. Pd, Ga, and $\mathrm{Ga}_{5} \mathrm{Pd}$ phase in PMMA matrix were identified by XRD. The characteristic absorption peak at $200 \mathrm{~nm}$ for nano-Ga/PMMA polymer solution, at $209 \mathrm{~nm}$ for nano-Pd/PMMA polymer solution were proved by UV-Vis; the binding energy changes of O1s, $\mathrm{Ga} 2 \mathrm{p} 3, \mathrm{Ga} 2 \mathrm{~d}$, and Pd3d were characterized by means of X-ray photoelectron spectroscopy. It is concluded that nano-Ga $5 \mathrm{Pd}$ was produced based on segment electronics shifting from Gallium to Palladium, and coordination was formed on segment electronics from Gallium to oxygen of PMMA ester group. The anisotropism ordered assembly of PMMA around nano-Ga-Pd particles were illuminated by transmission electron microscopy; it is further interpreted that nano Ga-Pd particles had ordered-assembly induced effect.
\end{abstract}

\section{Introduction}

Nanocomposite materials have increasingly become a major part of new synthetic materials all over the world owing to their applications in mechanics, tribology, optics, magnetism, and electronics $[1,2]$. Nanoalloy materials, which are different from the general alloy in both size and structure, have been one of the most promising and emerging research areas in advanced materials because of the unique properties $[3,4]$. Lee and Shingu [5] in Tokyo University firstly prepared nanoalloy materials by ball milling. Subsequently, lots of new methods were used to prepare nanoalloy materials $[6,7]$, such as chemical reduction. However, there existed limitations in those methods: the reaction system was more complicated and the properties of the as-synthesized nanoalloy became bad because of initiator or metal reductant used in the preparation process. And in those methods, the as-synthesized nanosized alloy materials conglomerated easily, and so the nano-effect of nanomaterials weakened. To resolve these problems, the idea of the organic-based nanometal came into being and new nanocomposite alloys were reported to possess interesting properties $[8,9]$.
Ultrasonic radiation method was used widely to prepare nanomaterials because of its convenience, emulsification, dispersion, and cavitation. Works on nanometal particles synthesis in emulsion polymerization without initiator by means of ultrasonic radiation were reported [10], but literature on preparing $\mathrm{Pd}-\mathrm{Ga}$ alloy nanocomposite particles by ultrasonic radiation cannot be found as far as our knowledge goes, let alone the ordered self-assembly of PMMAbased Pd-Ga nanocomposite. So-called self-assembly is the autonomous organization of components in system into patterns or structures without human intervention [11]. For polymer-based nanocomposites, the polymer chain would possess the ordered self-assembly phenomena due to nanoeffect of nanometal particles. South Korean scholars [12] had reported that on nano- $\mathrm{Fe}_{2} \mathrm{O}_{3}$ particles into composite would induce polystyrene-block-polypropylene (PS-b-PI) as composite matrix to self-assembly; the results showed that size and content of nano- $\mathrm{Fe}_{2} \mathrm{O}_{3}$ into the nanocomposites had great impact on ordered self-assembly structure of PSb-PI. Chinese scholars had studied that polyethylene (PE) crystallization would be induced by carbon nanotubes, of which the nanocomposites were composited [13]. XU group 
[14] preliminarily reported that ordered self-assembly of PMMA was induced by nano-Ag-Ga alloy. The ordered selfassembly with long-range orientation selectivity of PMMA induced by $\mathrm{Pd}-\mathrm{Ga}$ alloy is studied in the paper. As for ordered self-assembly of polymer into nanocomposites with nanometal particles, no paper was found as far as our knowledge goes.

In the paper, the PMMA-based nano Pd-Ga nanocomposites were prepared by ultrasonic radiation the gallium that has low-melting point $\left(29.78^{\circ} \mathrm{C}\right)$ is easily comminuted into nano-granules by ultrasonic radiation, and the nanoalloy composed of both gallium and Palladium is mutually formed by the nano-granules of gallium from the bulk liquid gallium and palladium clusters reduced from palladium salt solution without metal reductant. Meanwhile poly (methyl methacrylate) synthesized by ultrasonication without any initiator in the emulsion system was used to wrap the nanoalloy to prevent the nanoparticles from coagulating, so the Pd-Ga/PMMA nanoalloy composite particles with coreshell structure are prepared.

\section{Experimental}

Preparation of Pd-Ga/PMMA nanoalloy composite particles was carried out as follow. A block of gallium (purity: 99.99\%); high-purity palladium (II) chloride $\left(\mathrm{PdCl}_{2}\right)$ (Chenzhou Xiangchen Hi-tech Industry Co.Ltd, China), and a little of sodium dodecyl sulfate (abbreviated as SDS, purity: $90.0 \%$ ) were added into a beaker containing a proportional distilled water, then a proportional methyl methacrylate, which was supplied by Shanghai Chemical Reagent Company and washed with 5\% aqueous sodium hydroxide and subsequently washed by distilled water to neutrality, was poured into the beaker. Then, $\mathrm{N}_{2}$ was piped into the mixture in the beaker to get rid of dissolved oxygen in the mixture for $15 \mathrm{~min}$. The output power of the ultrasonic was adjusted to work efficiently before the ultrasonic generator (JY98III ultrasonic generator, Ningbo Scientz Biotechnology Co., Ltd., China, frequency: $20 \mathrm{KHz}$, output power: $200 \sim 1200 \mathrm{~W}$ ) was on. The reaction temperature was controlled between $40 \sim 45^{\circ} \mathrm{C}$ by circulating water. The system became emulsion and the color of the system became dusk after the ultrasonication for several minutes. The ultrasonic generator was terminated when the emulsion polymerization of methyl methacrylate and reduction of the palladium ion (II) were finished. The emulsion was refrigerated to demulsificate, then the precipitate was treated by filtering, washing, and drying in vacuum, and finally PMMA-based nano Pd-Ga composite particles were obtained.

The morphology of the nanoparticles was observed by means of high-resolution transmission electron microscope (TEM) (JEOL-2010, Japan Electronics Co., Ltd.) when a little of the powder samples was dispersed in acetone to form dispersion system, and the suspension was dropped to a copper grid. The crystalline phase of the powder was determined by using an X-ray diffractometer (XD-3, Beijing Pur-kinje General Instrument Co. Ltd. China) with DX$2000\left(\lambda_{\mathrm{CuK \alpha}}=0.15418 \mathrm{~nm}\right)$, using graphite-monochromatic

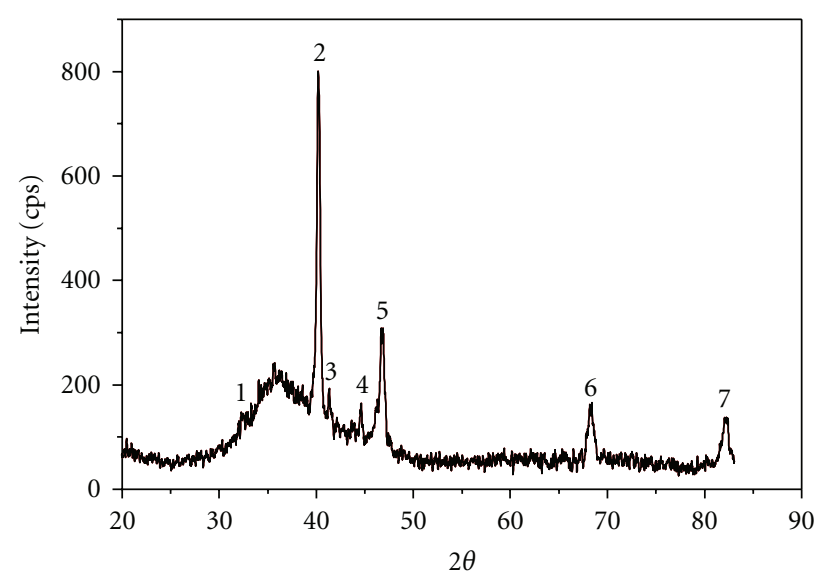

FIgURE 1: XRD pattern of the Pd-Ga/PMMA nanocomposite.

TAble 1: Values of $2 \theta$ and $d$ at XRD peaks of Pd-Ga/PMMA nanocomposite.

\begin{tabular}{lccccccc}
\hline Peak & 1 & 2 & 3 & 4 & 5 & 6 & 7 \\
\hline $2 \theta\left(^{\circ}\right)$ & 32.30 & 40.31 & 41.37 & 44.48 & 46.77 & 68.31 & 82.23 \\
$\mathrm{~d}(\mathrm{~nm})$ & 2.7691 & 2.2354 & 2.1809 & 2.0355 & 1.9402 & 1.3717 & 1.1712 \\
$\begin{array}{l}\text { Standard } \\
\mathrm{d}(\mathrm{nm})\end{array}$ & 2.7740 & 2.2407 & 2.1830 & 2.0410 & 1.9405 & 1.3721 & 1.1701 \\
\hline
\end{tabular}

$\mathrm{Cu}-\mathrm{Ka}$ radiation $(r=0.154 \mathrm{~nm})$ with an accelerating voltage of $40 \mathrm{KV}$, at scanning rate of $0.02^{\circ} \mathrm{S}^{-1}$ in $2 \theta$ ranging from $10^{\circ}$ to $85^{\circ}$. The UV spectrum of the samples were tested by TU-1901 dual-beam UV spectrophotometer (Beijing PUXI Co. Ltd in China). The chemical composition of Pd-Ga/PMMA nanocomposites was tested by Thermo ESCA-LAB 250 X-ray photoelectron spectroscopy (Thermo Corporation, American).

\section{Results and Discussion}

The infrared spectrum of the nanocomposites was collected after purification and pellet pressed together with potassium bromide, characteristic peak of $\mathrm{C}=\mathrm{C}\left(1638 \mathrm{~cm}^{-1}\right)$ nearly disappeared in $\mathrm{Pd}-\mathrm{Ga} / \mathrm{PMMA}$ spectrum, which indicated the polymerization of MMA monomer occurred completely by ultrasonic radiation. Characteristic peak of $\mathrm{C}=\mathrm{O}$ and $\mathrm{C}-\mathrm{H}$ in the spectrum of PMMA was shifted to short wave region in that of the nanocomposites, which hinted that there existed interaction between nanoalloy particles with PMMA matrix to some degree.

The X-ray diffraction patterns of Pd-Ga/PMMA composite particles by ultrasonic irradiation were showen in Figure 1; the four strong characteristic peaks, shown as peaks 2, 5, 6, and 7 in Figure 1, of Pd-Ga/PMMA composite lied in $2 \theta=40.31,46.77,68.31$, and 82.23, which were corresponding to crystal face of [111], [200], [220], and [311] of Pd (PCPDFWIN\#870639). The peaks at $2 \theta=32.30,41.37$, and 44.48, respectively, belonged to [211], [213] and [310] of $\mathrm{Ga}_{5} \mathrm{Pd}$ (PCPDFWIN\#15-0577). But the peak of [114] 


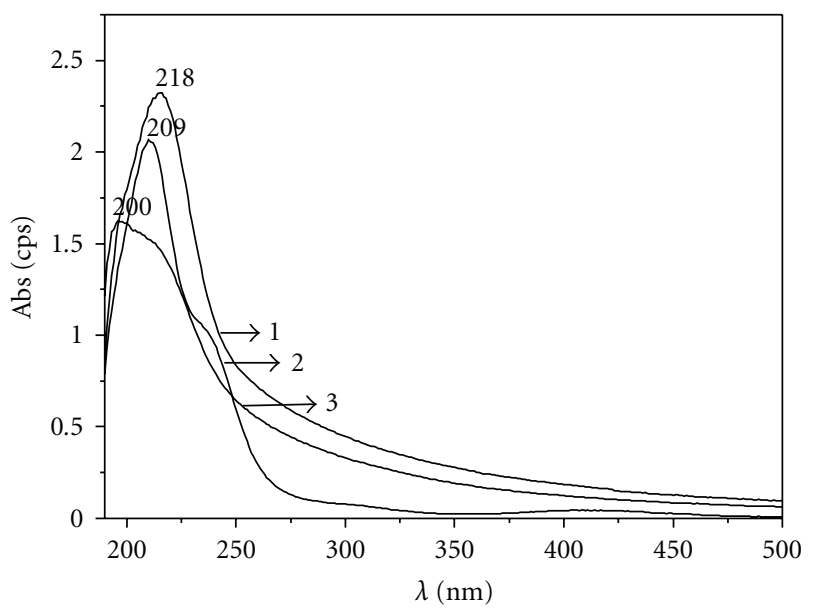

Figure 2: UV-Vis spectra of Pd-Ga/PMMA), nano-Pd/PMMA, and Ga/PMMA composites. (1) Pd-Ga/PMMA; (2) Pd/PMM; (3) Ga/PMMA.

(standard d values are $2.1960 \mathrm{~nm}$ ) of $\mathrm{Ga}_{5} \mathrm{Pd}$ was not found, it is possibly explained that the peak of [114] was overlapped by that of [111] of $\mathrm{Pd}$. The peak appeared at $2 \theta=35^{\circ}$ was of amorphous peak of $\mathrm{Ga}$ with the low-melting point.

UV-visible absorption spectra of the samples are diagrammed in Figure 2. The peak that appeared at $207 \mathrm{~nm}$ was from Ga/PMMA composite, the peak at $209 \mathrm{~nm}$ from nano-Pd/PMMA composite, and the peak at $218 \mathrm{~nm}$ from nano $\mathrm{Pd}-\mathrm{Ga} / \mathrm{PMMA}$ composite. It was obvious that curve 3 at $218 \mathrm{~nm}$ for nano Pd-Ga/PMMA composite was different from curve 1 at $207 \mathrm{~nm}$ and curve 2 at $209 \mathrm{~nm}$ in absorption position and strength; it was indicated that this was contributed from palladium gallium alloy $\mathrm{Ga}_{5} \mathrm{Pd}$ as dispersing nano-phase into nanocomposites.

To further investigate the microstructure information of the nano Pd-Ga/PMMA composites, XPS of the nano $\mathrm{Pd}-\mathrm{Ga} / \mathrm{PMMA}$ composite was performed and shown as in Figure 3(a), the results suggested that there were binding energy peaks of elements of carbon, oxygen, palladium, and gallium. The peaks of Ga2p region of the gallium was shown as Figure 3(b), the peak positioned at $1118.81 \mathrm{eV}$ was correspondant to $\mathrm{Ga} 2 \mathrm{p} 3$. In comparison with pure gallium with standard energy spectrum peaks at $1116.6 \mathrm{eV}$ of Ga2p3 electron, the binding energy peaks were shifted to high region, increasing $2.2 \mathrm{eV}$. The peaks of Ga3d region of the gallium were shown as in Figure 3(c); the peaks positioned at $20.64 \mathrm{eV}$ and $18.02 \mathrm{eV}$, respectively, were correspondant to $\mathrm{Ga} 3 \mathrm{~d} 3 / 2$ and $\mathrm{Ga} 3 \mathrm{~d} 5 / 2$, in comparison with pure gallium with standard energy spectrum peaks at $1116.6 \mathrm{eV}$ of Ga2p3 electron. At $2138 \mathrm{eV}$ of $\mathrm{Ga} 3 \mathrm{~d} 3 / 2$ and $18.52 \mathrm{eV}$ of $\mathrm{Ga} 3 \mathrm{~d} 5 / 2$, respectively, the binding energy peaks were shifted to high region, increasing $2.2 \mathrm{eV}, 0.74 \mathrm{eV}$ and $0.5 \mathrm{eV}$, respectively; this phenomenon indicated that the chemical environment around $\mathrm{Ga}$ atoms was changed; the binding energy within the electronic shell was increased because of the decrease of valence electron density and the shielding effects of domestic electronic shell. In Figure 3(c), the energy spectrum peaks of $\mathrm{Pd} 3 \mathrm{~d}$ electrons positioned at $335.43 \mathrm{eV}$, which were dropped down $2.07 \mathrm{eV}$ than the standard energy spectrum peaks of $\mathrm{Pd} 3 \mathrm{~d}$ electrons that were positioned at $337.5 \mathrm{eV}$, that is, the energy spectrum peaks of $\mathrm{Pd} 3 \mathrm{~d}$ electrons within nanocomposites, moved to the low-binding energy region. This illustrated that chemical environment of palladium was changed due to getting some electronics from other atoms. For binding energy change of the gallium atom, on the one hand, in terms of interaction between gallium and palladium, the segment electron in $4 \mathrm{~d}$ orbital of gallium flowed directly to $5 \mathrm{~s}$ orbital of palladium in order to form a new alloy, namely, $\mathrm{Ga}_{5} \mathrm{Pd}$ generated from $\mathrm{Pd}$ with $\mathrm{Ga}$; on the other hand, coordination energy was based on segment electronics shifting from gallium to oxygen attached to ester group of PMMA into nanoalloy/PMMA composite. The curve of XPS spectrum of O1s pertain to the nano Pd-Ga/PMMA composite was fitted by means of Origin 6.1 shown in Figure 3(e); curve 1 peaked at $531.62 \mathrm{eV}$, in Figure 3(e) was originated from carboxyl $(\mathrm{C}=\mathrm{O})$, curve 2 peaked at $533.39 \mathrm{eV}$ from ester group (C-O-C), and curve 3 from integration fitting peak of O1s in the two chemicals environment. Based on the standard dates of $(532.62 \mathrm{eV})$ carboxyl $(\mathrm{C}=\mathrm{O})$ and $(533.39 \mathrm{eV})$ ester group $(\mathrm{C}-\mathrm{O}-\mathrm{C})$, the $\mathrm{O} 1 \mathrm{~s}$ binding energy peaks shift to lower region, which were reduced to $0.68 \mathrm{eV}$ and $0.51 \mathrm{eV}$, respectively; it was probably due to carbonyl oxygen atom accepting electronics cloud to from gallium atom form nanoalloy of $\mathrm{Pd}-\mathrm{Ga}$ into the nanocomposite. The XPS results proved that there existed interaction between carboxyl $(\mathrm{C}=\mathrm{O})$ oxygen and the nanoalloy.

The morphology of the nano Pd-Ga/PMMA composites was showen in Figure 4; it demonstrated that the nanocomposite was small in size about $\sim 25 \mathrm{~nm}$ with nanometal particles as core and PMMA as shell. The interesting TEM images of the nanocomposites were formed when observed in high-resolution TEM; it hinted that there were ordered self-assembly of PMMA in addition to possessing in ordered distribution state around the nanoalloy in Figure 4; the ordered self-assembly of PMMA possibly had something to do with not only content of nanometal, but also distance and direction apart from surface of nanometal in distribution. The more the content of the nanometal is, the more the obvious is phenomenon of ordered self-assembly of PMMA, comparing Figure 4 (a) based on metal content $0.15 \%$ with Figure 4 (b) based on metal content $0.3 \%$. For selectively of distance and direction apart from surface of nanometal in distribution, on the one hand, ordered direction of PMMA self-assembly was vertical near to the surface of nanometal particles; on the other hand, ordered direction of PMMA self-assembly was changed from vertical to slope even to horizontal direction apart from the surface of the nanometal particles, shown as the inset in Figure 4(b). The mechanism of the ordered self-assembly of PMMA was probably imagined as induced effect of nanometal Pd-Ga particles into nanocomposites due to interaction of nano PdGa with PMMA illustrated above. Based on the interaction of palladium gallium nanocrystals as discontinuous phase with PMMA as continuous phase, why did nanometal contained gallium as one of components into polymer-based nanocomposites would occur the ordered self-assembly, the 


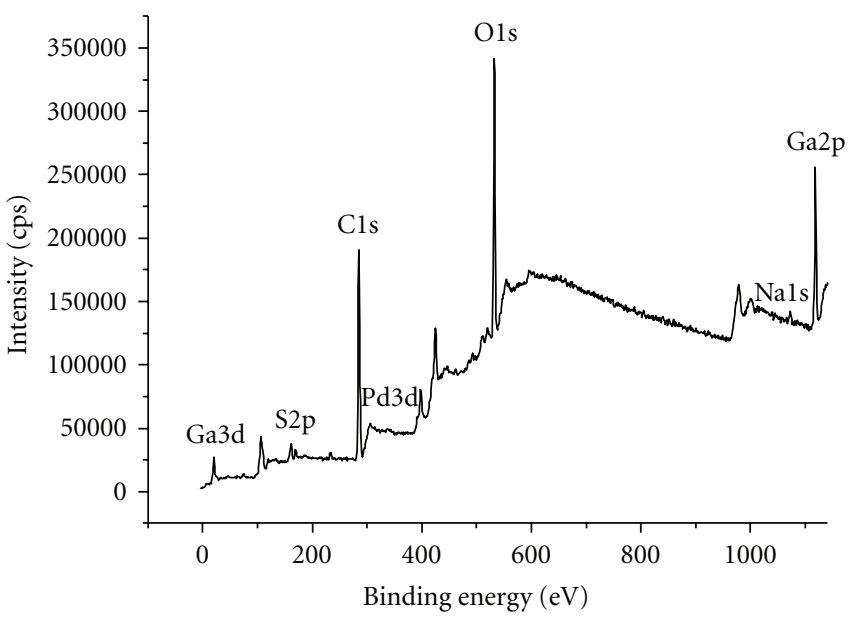

(a)

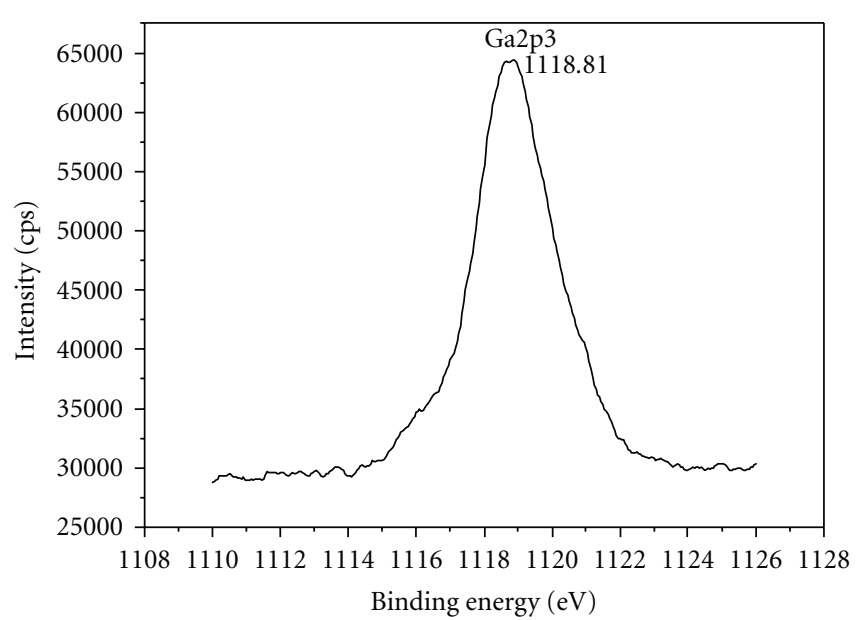

(b)

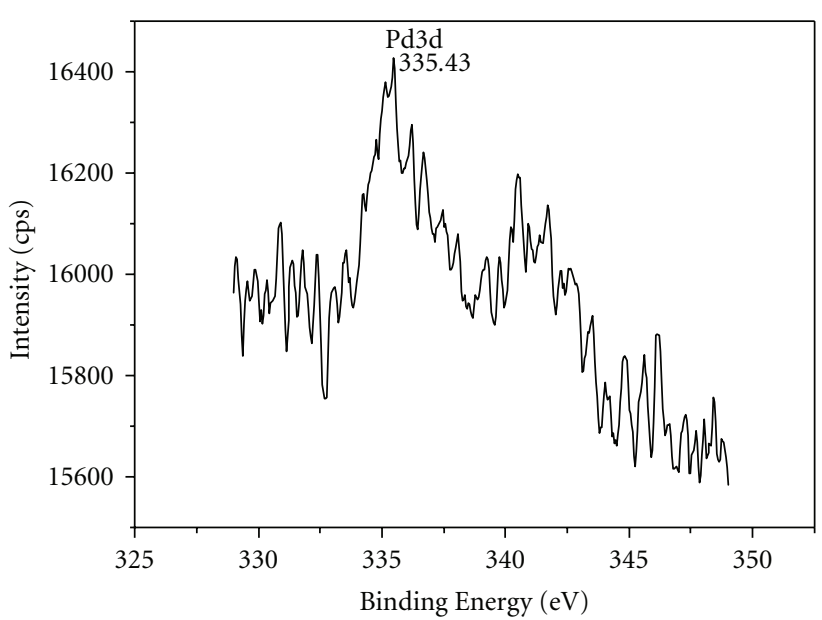

(d)

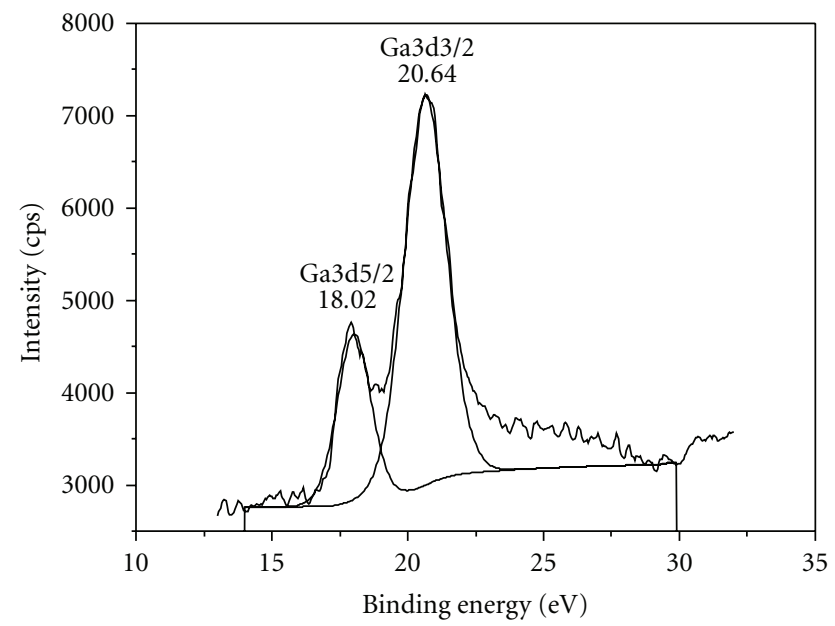

(c)

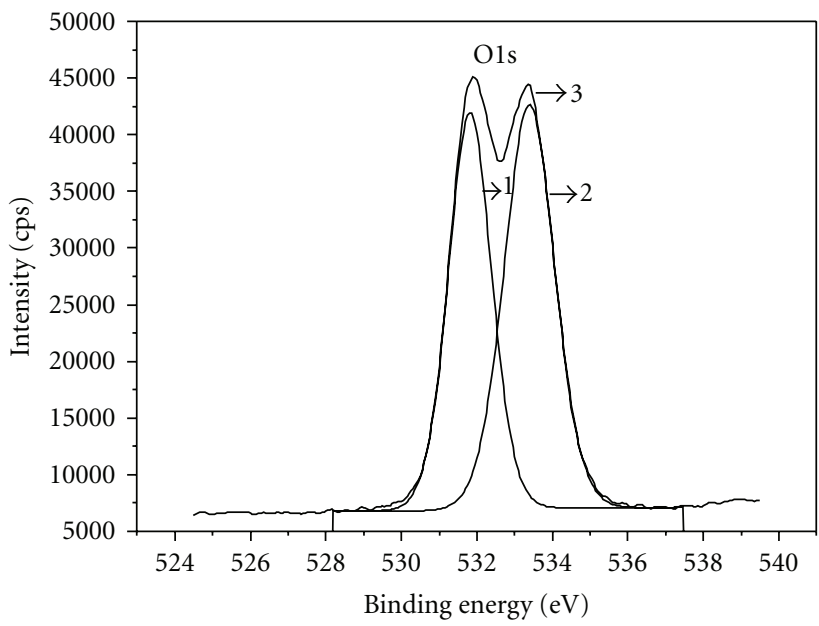

(e)

Figure 3: XPS of nano-Ga-Pd/PMMA composites powder. (a) XPS survey spectrum of nanocomposites; (b) Ga2p; (c) Ga3d; (d) Pd3d; (e) fitting curve of O1s. (1) $\mathrm{C}=\mathrm{O}$; (2) $\mathrm{C}-\mathrm{O}-\mathrm{C}$; (3) integrate fitting peak of $\mathrm{O} 1 \mathrm{~s}$ in the two chemicals environment. 


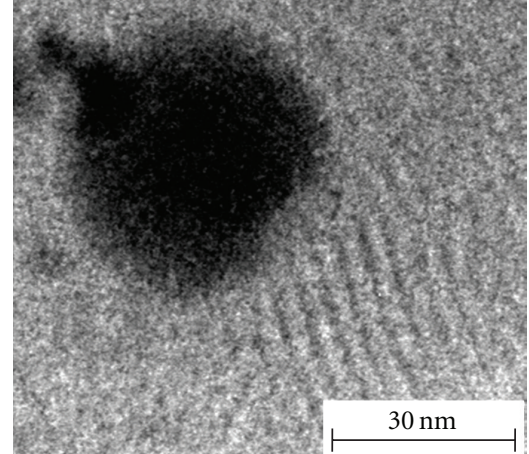

(a)

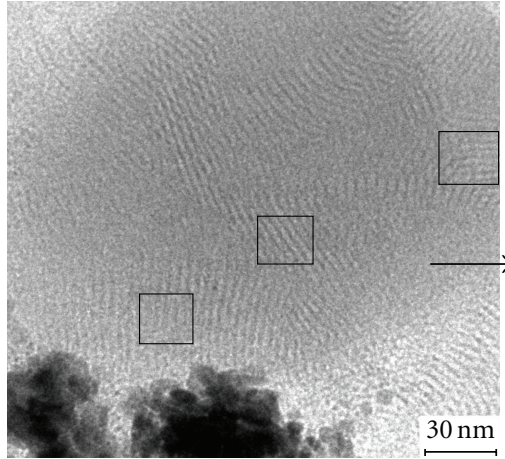

(b)

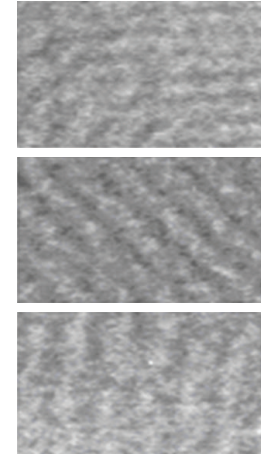

FIgure 4: TEM images of the Pd-Ga/PMMA nanocomposite. (a) Metal Contention $0.15 \%$ (b) Metal Contention $0.3 \%$.

possibly reason was suggested to come from stable atomchain structure of gallium. Gallium itself possessed property to form stable atom-chain structure [15], and exhibit the most stable " $\mathrm{Z}$ " configuration. Gallium was mainly a component in the $\mathrm{Ga}_{5} \mathrm{Pd}$ nanoalloy and was distributed possibly on the surface of the nanoalloy. On coordination of gallium atoms with oxygen of ester group attached to PMMA, oxygen atoms attached to PMMA were arranged along the direction of gallium atom-chain structure as a template; so, there were ordered oxygen atoms array and then ordered self-assembly of PMMA as polymer continuous phase into nanocomposites. The ordered self-assembly of PMMA as continuous phase into nano-Ag-Ga/PMMA composites possessed isotropic phenomenon in reported paper by XU group [14], which was closed ringlike around AgGa particles as dispersing phase into nanocomposites; it was completely different to that of PMMA from the Pd-Ga nanocomposites. The possible reason was suggested that the nanosilver gallium alloy into polymer-based nanocomposites was presented to be isotropic based on hexagonal crystal, whereas the nano-palladium gallium alloy into polymerbased nanocomposites did not, it was found to be antitropic based on tetrahedron crystal.

\section{Conclusions}

Nano-Ga-Pd /poly methyl methacrylate (PMMA) composite materials were prepared with the palladium chloride solution containing metal gallium without initiator reducer, MMA as monomer, and sodium dodecyl sulfate (SDS) as emulsifier under ultrasonic generator, in which $\mathrm{Pd}^{2+}$ ion was reduced to nano-palladium particles, meanwhile produced $\mathrm{Ga}_{5} \mathrm{Pd}$ with microdrop of liquid gallium, and the monomers were polymerized simultaneously by ultrasonic radiation. The structure of nanocomposite with nano metal was characterized by means of IR, XRD, UV-Vis, TEM, and XPS. Pd, and $\mathrm{Ga}_{5} \mathrm{Pd}$ as dispersing phase in PMMA matrix was identified by XRD. The results of UV-Vis spectrum showed that there was a characteristic absorption peak at $200 \mathrm{~nm}$ for nano$\mathrm{Ga} / \mathrm{PMMA}$ polymer solution, at $209 \mathrm{~nm}$ for nano-Pd/PMMA polymer solution, and at $218 \mathrm{~nm}$ for nano Pd-Ga/PMMA;
XPS proved that the O1s binding energy of $\mathrm{C}=\mathrm{O}$ and $\mathrm{C}-$ $\mathrm{O}-\mathrm{C}$ into nanocomposite was decreased compared to that of the standards, respectively, the meanwhile binding energy of Ga2p3, Ga2d3/2, and Ga2d5/2 into Pd-Ga nanoalloy of the nanocomposite were increased; however, Pd3d binding energy was decreased; it is concluded that nano $\mathrm{Ga}_{5} \mathrm{Pd}$ was formed on shifting of segment electronics of Gallium to Palladium, and coordination of gallium attached to nano Pd-Ga alloy and oxygen of ester group attached to PMMA was found on shifting of segment electronics of gallium to oxygen. So, there was interaction of nano metal particles with the polymer as matrix. Based on the interaction, TEM illuminated that ordered self-assembly of PMMA as continuous phase around nano-Ga-Pd particles into nanocomposites was presented and was found to be of anisotropism; it was further deduced that nano-Ga-Pd particles possessed induced effect on ordered self-assembly of polymer.

\section{Acknowledgments}

The authors are grateful for the support of the Natural Science Foundation of Anhui Province (Grant 090414183) and Science Foundation of Education, Department of Anhui Province, China (Grant KJ2009A46).

\section{References}

[1] R. Ferrando, J. Jellinek, and R. L. Johnston, "Nanoalloys: from theory to applications of alloy clusters and nanoparticles," Chemical Reviews, vol. 108, no. 3, pp. 845-910, 2008.

[2] H.-J. Chen, Z.-W. Li, B.-B. Hu et al., "Preparation of Bi-PbSn-Cd nanoalloy particles and its tribological properties as an additive in liquid paraffin," Mocaxue Xuebao/Tribology, vol. 25, no. 2, pp. 169-172, 2005.

[3] H. P. Wang, C.-Y. Liao, F.-L. Chen, C.-H. Huang, and Y. Fukushima, "Applications of $\mathrm{Cu@C} \mathrm{Nanoparticles} \mathrm{in} \mathrm{new} \mathrm{dye-}$ sensitized solar cells," Journal of Nanomaterials, vol. 2009, Article ID 698501, 4 pages, 2009.

[4] A. Das, R. Jurk, K. W. Stöckelhuber et al., "Nanoalloy based on clays: intercalated-exfoliated layered silicate in high performance elastomer," Journal of Macromolecular Science, Part A, vol. 45, no. 2, pp. 144-150, 2008. 
[5] K.-M. Lee and P. H. Shingu, "Solid state reaction between powders and foils by low-energy ball milling," Journal of Alloys and Compounds, vol. 241, no. 1-2, pp. 153-159, 1996.

[6] H. Kim and J. Cho, "Synthesis and electrochemical properties of $\mathrm{Sn}_{87} \mathrm{Co}_{13}$ alloys by $\mathrm{NaBH}_{4}$ and sodium naphthalenide reduction methods," Electrochimica Acta, vol. 52, no. 12, pp. 4197-4201, 2007.

[7] H. Chen, Z. Li, Z. Wu, and Z. Zhang, "A novel route to prepare and characterize Sn-Bi nanoparticles," Journal of Alloys and Compounds, vol. 394, no. 1-2, pp. 282-285, 2005.

[8] G. C. Xu, L. X. Xing, L. J. Ji, J. S. Shi, and M. Z. Zhang, "Synthesis and assembly of nano-copper/PMMA-b-PSt by ATRP," Journal of Thermoplastic Composite Materials, vol. 22, no. 5, pp. 475-484, 2009.

[9] W. A. Lopes and H. M. Jaeger, "Hierarchical self-assembly of metal nanostructures on diblock copolymer scaffolds," Letters to Nature, vol. 414, no. 6865, pp. 735-738, 2001.

[10] G. C. Xu, J. Y. Xiong, X. L. Ji, and Y. L. Wang, "Synthesis of nanosilver/PMMA composites via ultrasonically Bi-in situ emulsion polymerization," Journal of Thermoplastic Composite Materials, vol. 20, no. 6, pp. 523-533, 2007.

[11] J. Ming, A. Eisenberg, L. Guojun, and Z. Xi, Macromolecules Self-Assembly, Science press, Beijing, China, 2006.

[12] M. J. Park, J. Park, T. Hyeon, and K. Char, "Effect of interacting nanoparticles on the ordered morphology of block copolymer/nanoparticle mixtures," Journal of Polymer Science, Part B, vol. 44, no. 24, pp. 3571-3579, 2006.

[13] L. Li, C. Y. Li, and C. Ni, "Polymer crystallization-driven, periodic patterning on carbon nanotubes," Journal of the American Chemical Society, vol. 128, no. 5, pp. 1692-1699, 2006.

[14] J. Zhang, G. Xu, Y. Wang, and W. Chen, "Characterization of the structure of Ag-Ga/PMMA nanoparticles," Acta Materiae Compositae Sinica, vol. 24, no. 4, pp. 67-70, 2007.

[15] S.-Q. Wu, G.-Z. Chen, and Z.-Z. Zhu, "Structural stabilities and electronic structures of $\mathrm{Ga}$ atomic chains," Chinese Journal of Chemical Physics, vol. 19, no. 3, pp. 219-222, 2006. 

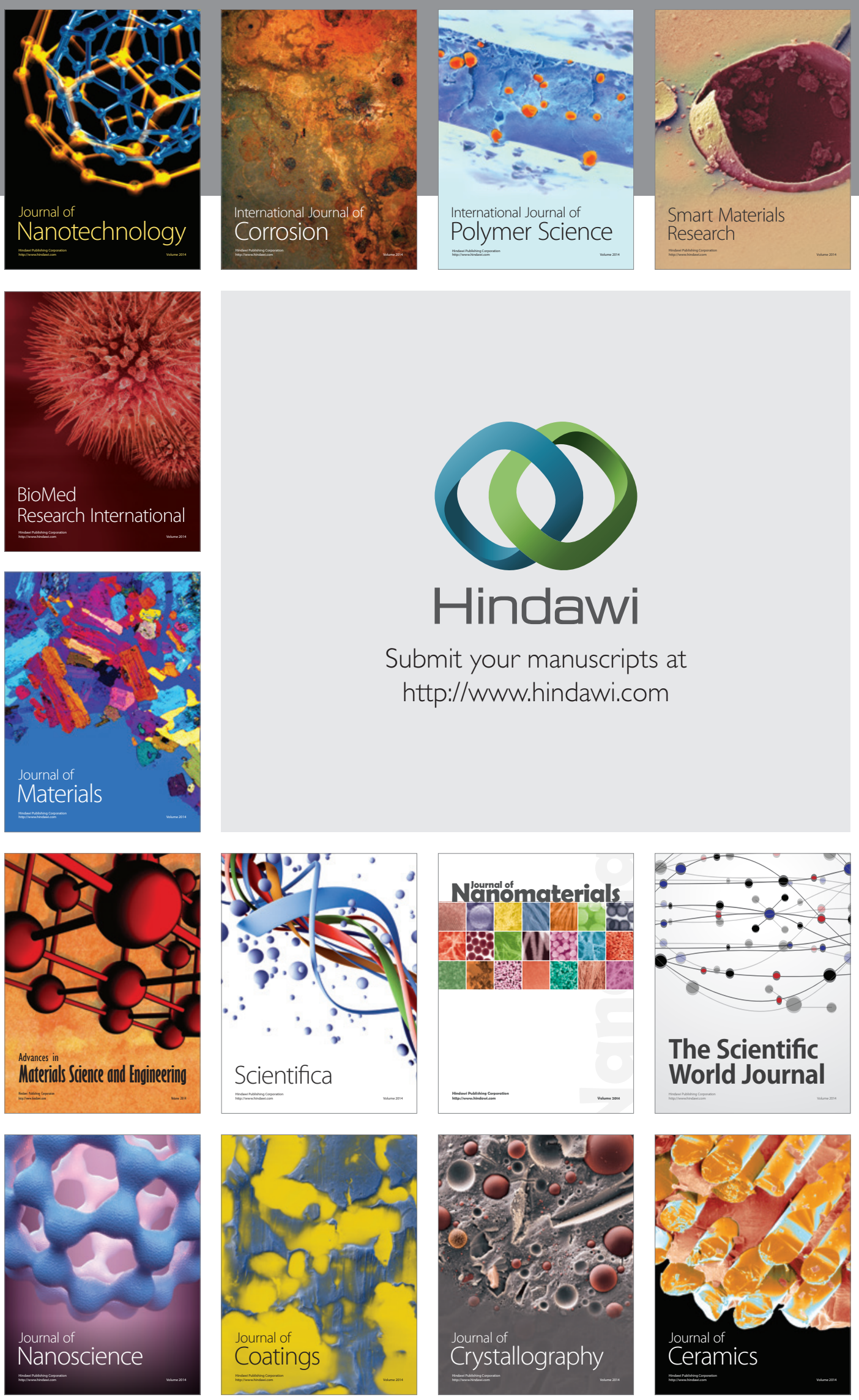

The Scientific World Journal

Submit your manuscripts at

http://www.hindawi.com

\section{World Journal}

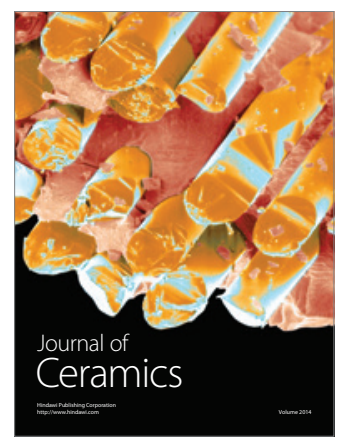

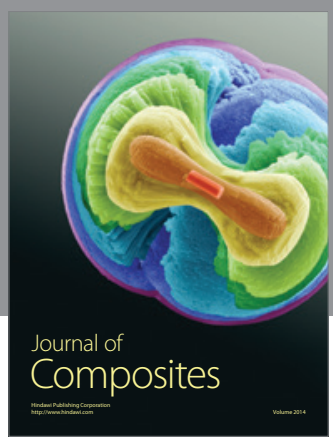
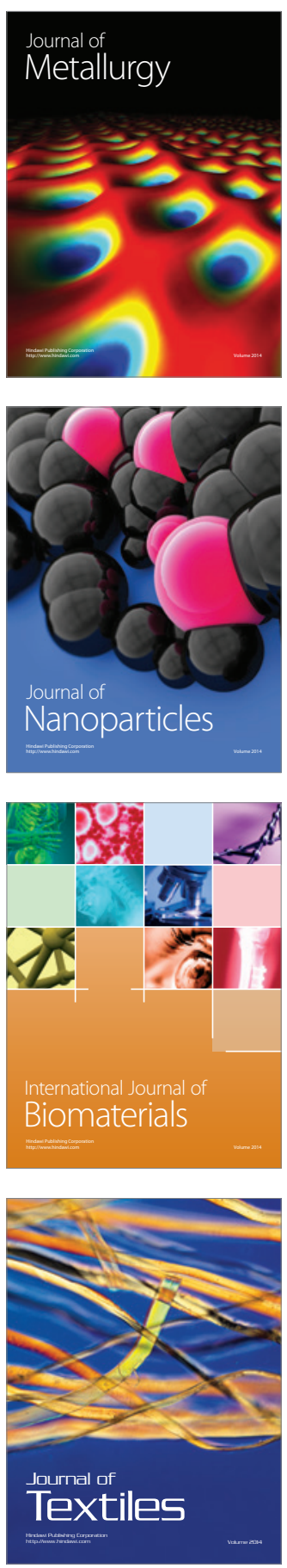